\title{
Development of Pressure Sensor for Oil and Gas Field Based on Sputtered Thin Film
}

\author{
Yong Chen ${ }^{1, a^{*}}$, Shuangquan $\mathrm{Liu}^{2, \mathrm{~b}}$ and Xiaohong Bai ${ }^{1, \mathrm{c}}$ \\ ${ }^{1}$ Oil \& Gas Technology Research Institute, Changqing Oilfield Company, \\ Xi'an,Shaanxi710018,China \\ ${ }^{2}$ Low-permeability oil and gas exploration and development of National Engineering Laboratory, \\ Xi'an,Shaanxi710018,China \\ a187055127@qq.com, ${ }^{\mathrm{b}}$ Isq_cq@126.com, ${ }^{\mathrm{c} b x h}$ _cq@126.com
}

\begin{abstract}
Keywords: oil and gas field; pressure sensor; sputtered thin film
Abstract. Gas well head pressure sensors are the important front-end equipments of digitalization construction of Changqing oil field. Foreign pressure sensors are once relied on for a long time there. A problem urgent to be solved of digitalization construction is to develope pressure sensors with independent intellectual property and with integrated advantages of high precision, high stability, high reliability and wide temperature range. This paper introduced the development of a kind of pressure sensor based on technology of sputtered thin film and method of micro-machining. Design and manufacture of the pressure-sensitive element are introduced, which are comprised of elastic body, insulating film, wire grid etc. Meanwhile, tests and experiments are developed. The pressure sensors this paper developed possess the merits of high precision, high stability, and could meet the long term pressure testing requirements of oil and gas field.
\end{abstract}

\section{Introduction}

With an area of 370 thousands square kilometer, the Ordos basin stretch across five provinces of Shaanxi, Gansu, Ningxia, NeiMonggol and Shanxi, and nature environments are adverse there. In recent years, some large gas fields have been probed in the Ordos basin, namely, Jingbian gas field, Yulin gas field and Sulige gas field [1]. With the enlarging of devoloping scale of gas field, more and more production wells will be constructed. Digital management must be relied to supervise more than ten thousands of wells effectively [2]. Sensors are the most fundamental and important link of digitalization.

Pressure is the soul of gas field development. Gas well head pressure data is important for gas field management such as safety, production and performance analysis, etc. Currently, high performance pressure sensors in oil and gas field in china mainly depend on import, which seriously restrict the improvement of petrochemical industry [3].

Pressure sensors this paper introduced manufacture inner pressure-sensitive elements by using method of ion beam sputtering, and could work over $300 \square$, as well as the insulation resistance of $10^{6}$ $\mathrm{M} \Omega$ could be achieved at $500 \mathrm{~V}$ DC. Meanwhile, it possess the merits of high precision, high stability, and could meet the long term pressure testing requirements of oil and gas field.

\section{Structure Design of Pressure-Sensitive Elements of Sputtered Thin Film Pressure Sensor}

The basic principle of this sputtered thin film pressure sensor is using the theory of the stress and deformation of metal elastic diaphragm. There are functional relationships between deformation of diaphragm and changes of value of resistance adhering to the diaphragm. Deformation amount of diaphragm could be obtained via mesuring changes of resistance values through electric bridge, and then pressure values corresponding will be acquired.

Periphery fixed round metallic film is used for elastic element, and is shown in Fig.1. Because that the maximum deflection of flat diaphragm is not more than one third of film thickness, small deflection 
theory is used. Pressure act on the inner surface of flat diaphragm uniformly. Under the condition above, radial strain distribution graph of flat diaphragm is shown in Fig.2.

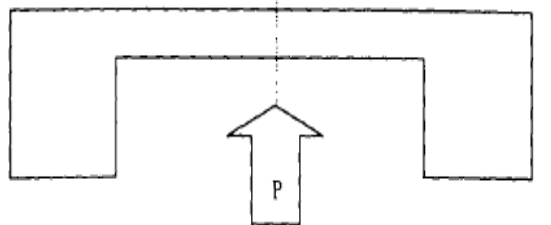

Fig.1 Periphery fixed round metallic film

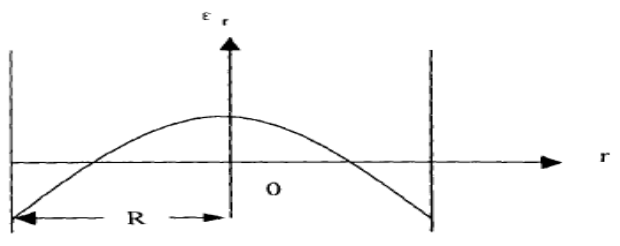

Fig.2 Radial strain distribution graph

Radial strain as following:

$$
\varepsilon_{r}=\frac{3 p\left(1-\mu^{2}\right)}{8 E h^{2}}\left(R^{2}-r^{2}\right)
$$

Where $\mathrm{p}$ is uniformly distributed pressure; $\mathrm{h}$ is film thickness; $\mathrm{R}$ is radius of film; $\mathrm{r}$ is any site radius of film; $\mu$ is Poisson ratio of film material; $E$ is elastic modulus of film material.

At centre of the film, tangential strain is equal to radial strain, and positive maximum occurs.

$$
\varepsilon_{r 0}=\varepsilon_{v 0}=\frac{3 p R^{2}}{8 E h^{2}}\left(1^{2}-\mu^{2}\right)
$$

Deflection of flat diaphragm as following:

$$
w=\frac{3 p\left(1-\mu^{2}\right)}{16 E h^{3}}\left(R^{2}-r^{2}\right)^{2} \text {. }
$$

At centre of the film, maximum deflection as following:

$$
w_{0}=w_{\max }=\frac{3 p\left(1-\mu^{2}\right) R^{4}}{16 E h^{3}}
$$

By using the technology of semiconductor photolithography and ion beam sputtering, sensitive resistance could be made very small and mainly be arranged at edge or center of the elastic diaphragm. Hence, radial strain is mainly considered. Strain distribution graph of flat diaphragm via finite element method through ANSYS is shown in Fig.3. It accord with the theoretical calculation.

According to the result of analysis, that designing the sensitive wire grid reasonably could improve the sensitivity of the sensor and ameliorate the nonlinearity as well.

Import data to COMSOL after modeling through SolidWorks. Then the solution region is given. Material is nichrome, and conductivity $\sigma$ is $8.06451 \times 105 \mathrm{~S} / \mathrm{m}$. Give the corresponding boundary conditions reasonably, and the result of potential distribution graph is shown in Fig.4.

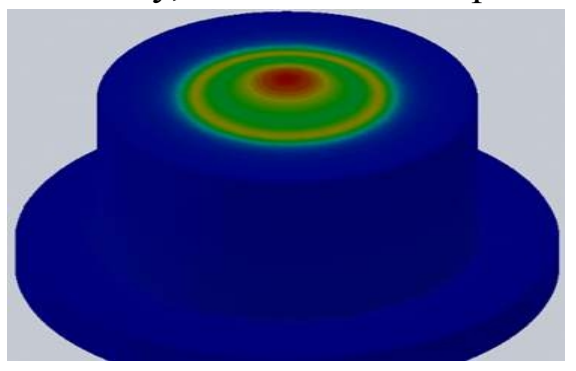

Fig.3 Strain distribution of flat diaphragm

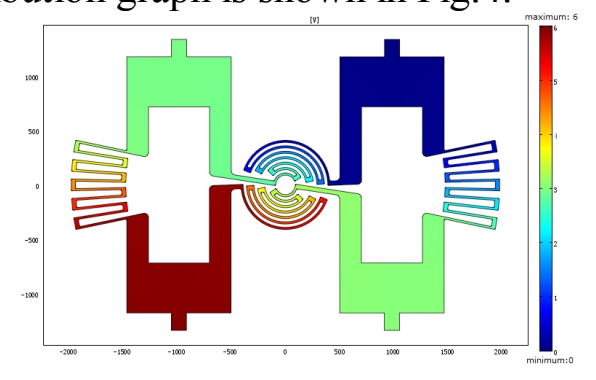

Fig.4 Potential distribution of sensitive wire grid

The width and length of the sensitive wire grid designed could be adjusted correspondingly according to the result of potential distribution graph. 


\section{Fabrication Process of Sputtered Thin Film Pressure Sensor}

Fabrication of Elastic Body Aiming at the characteristics of pressure testing environment of oil and gas field, such as high stability, impact resistance and corrosion resistance, substrates of elastic body are selected optimally. Thin film resistors of the sensor are deposited on the surface of elastic body. Hence, hysteresis and repeatability of the sensor would be under the influence of the characteristics of the elastic body material, such as after effect, creep, quality factor etc. So does full range drift of the sensor. From process point of view, material of elastic body must be easily to machining including grinding and polishing. Thus, a kind of special stainless steel namely 17-4PH is selected, which possess excellent quality of mechanical characteristic and higher corrosion resistance, and could meet the requirement of working under severe environment. Sensitive body this paper developed have small and exquisite structure and high precision, particularly, elastic beam thickness of which is merely $0.42 \mathrm{~mm}$.

Surface of the sensitive body must be grinded and polished during fabrication, and surface roughness must be less than $50 \mathrm{~nm}$. An innovative method is introduced, which combine weight grinding with pneumatic polishing, and improve the qualified rate by $50 \%$.

Fabrication of Insulating Film A key step of manufacturing sensitive body is the fabrication of insulating film which play a role of insulating alloy film from elastic body. Compact and defectless are required and insulation resistance must be more than $1000 \mathrm{M} \Omega$. If the value of insulation resistance dropped, current would outflow partly and led to the sensitivity decreasing significantly. The other adverse consequence of dropped insulation resistance is zero drift, which affect the long term stability of the sensor.

Material of $\mathrm{SiO}_{2}$ is adopted in fabrication of insulating film and accelerating voltage is $900 \mathrm{~V}$ when sputtering. Meanwhile, quality control and detection of the finished insulating film are pursued to ensure that the components are correct. The result of energy spectrum analysis is shown in Fig.5.

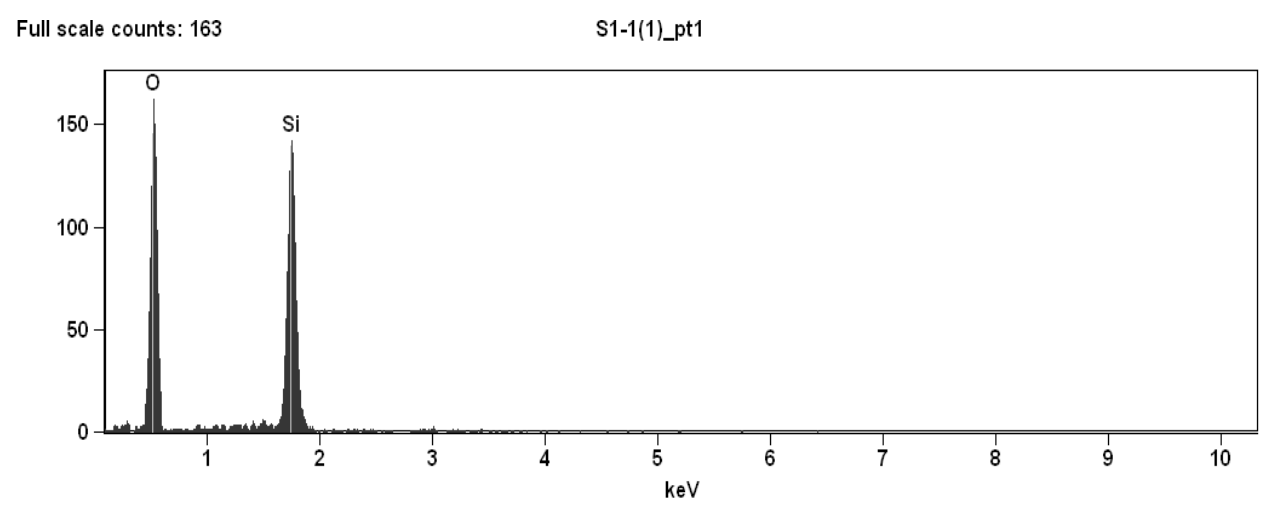

Fig.5 Energy spectrum analysis graph

According to the result of energy spectrum analysis, sputtered material of insulating film meet the requirement. On the basis of energy spectrum analysis, morphology of insulating film is observed by using electron microscope to confirm if the morphology be fit for the next process.

Fabrication of Sensitive Wire Grid The process of manufacturing sensitive wire grid include following steps:a) sputter sensitive resistive film on the elastic substrate; b) coat positive photoresist on sensitive resistive film; c) place strain graphic mask on positive photoresist and exposure; d) develop, fixing, and remove needless positive photoresist; e) etch unprotected resistive film by using ion beam sputtering; f) remove photoresist on the resistance grid. The diagrammatic sketch of the process of manufacturing sensitive wire grid is shown in Fig.6. 


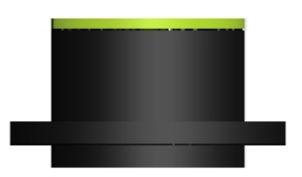

(a)

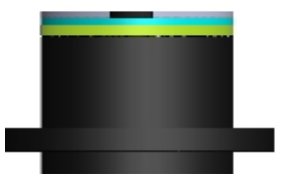

(c)

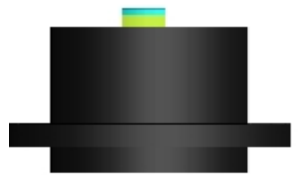

(e)

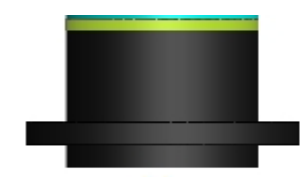

(b)

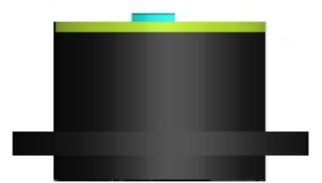

(d)

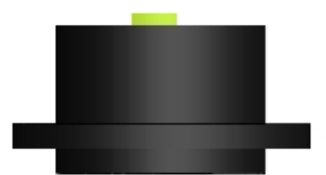

(f)

Fig.6 Process of manufacturing sensitive wire grid

Inspection must be made on the completed sensitive wire grid and one of the important procedures is to confirm if the surface of the grid could meet the requirement of sensors. Detecting picture of the wire grid is shown in Fig.7. Completed sensitive body of sputtered thin film pressure sensor is shown in Fig.8.

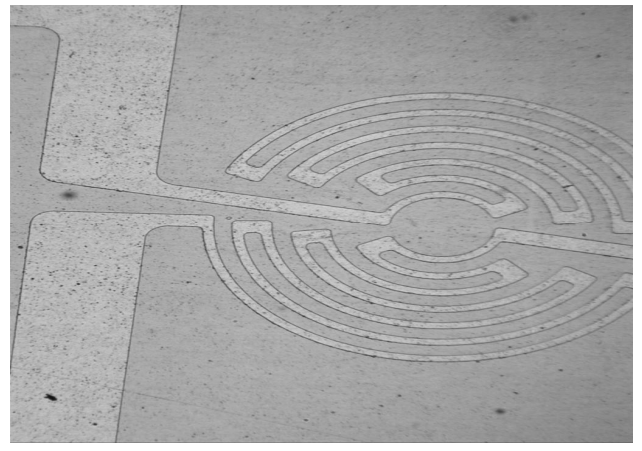

Fig.7 Detecting picture of the wire grid

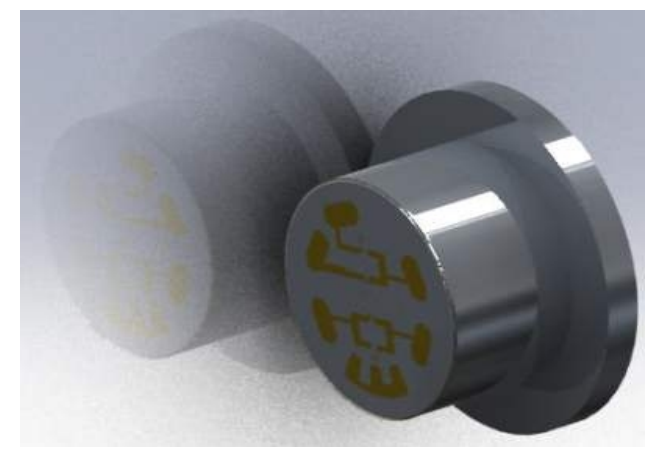

Fig.8 Completed sensitive body of sensor

\section{Testing Experiment of Sputtered Thin Film Pressure Sensor}

After integrate the sensitive body and pressure interface by laser welding, pressure sensitive component are obtained. Test item of the sensor mainly include insulation resistance, static calibration, and fatigue test etc.

Testing experiments of insulation resistance are developed by using insulation tester. Under the condition of keeping 2 minutes at $500 \mathrm{~V} \mathrm{DC}$, the maximum of insulation resistance of $10^{6} \mathrm{M} \Omega$ could be achieved. Meanwhile, static calibration tests of the sensor are carried out. According to the test, static parameters of the sensor are obtained as follow: Nonlinear is $0.08 \% \mathrm{FS}$, hysteresis is $0.07 \% \mathrm{FS}$, precision is $0.1 \% \mathrm{FS}$. Therefore, the gas well head pressure sensors designed possess a high performance.

To test and verify if the long-term stability of the sputtered thin film pressure sensor devoloped could meet the requirement of the field, fatigue tests are carried out. Loading pressure is $70 \%$ of the rated pressure with a pulsation of once every two seconds when being fatigue, and the total fatigue times is one million.

Result of fatigue test of zero point is shown Fig.9. Result of fatigue test of full-scale output is shown in Fig. 10. 


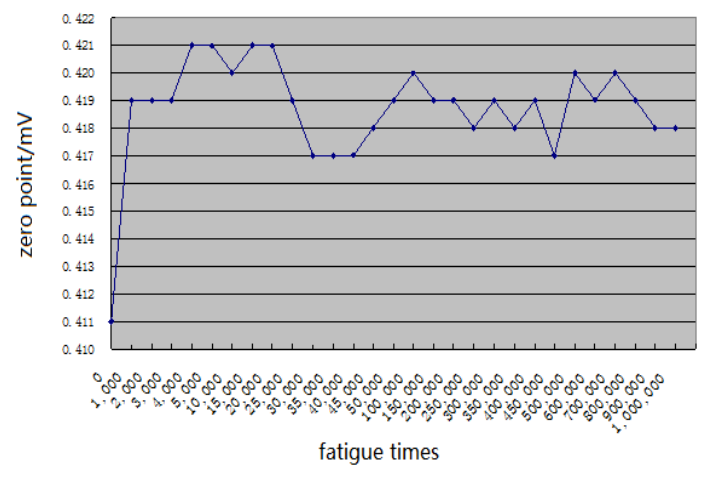

Fig.9 Fatigue test of zero point

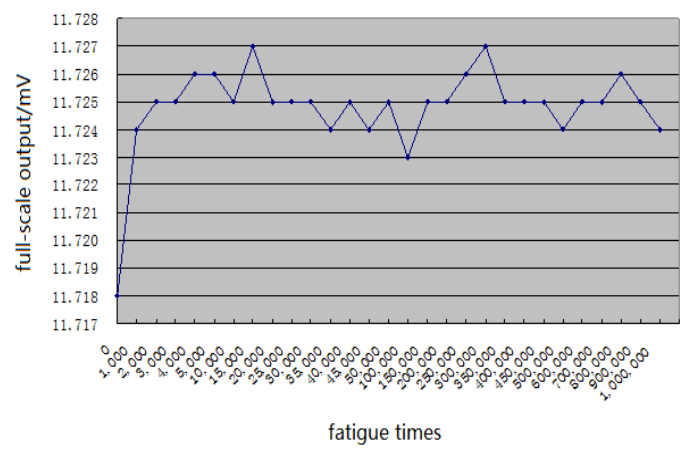

Fig.10 Fatigue test of full-scale output

According to the test result, zero point and full-scale output of the pressure sensitive component tend to be steady after 2000 times of fatigue. Variable quantities of the two parameters are in the range of requirement and the sensors have a good stability.

\section{Conclusions}

(1) The sputtered thin film pressure sensor this paper designed, which inner pressure-sensitive elements are manufactured by the method of ion beam sputtering, possess characteristics of high temperature resistance and could work over $300 \square$.Also, it has a good insulation strength and the insulation resistance of $10^{6} \mathrm{M} \Omega$ could be achieved at $500 \mathrm{~V}$ DC.

(2) Meanwhile, the sputtered thin film pressure sensors devoloped possess the merits of high precision, high stability, and could meet the long term pressure testing requirements of oil and gas field.

\section{Acknowledgements}

This work is supported by the National High Technology Research and Development Program (Project code: 2011AA040401). The authors appreciate the help of Senior Engineer Guanghong Gai from Shaanxi Electrical Institute for his support and thank Mr. Long Qi for his help with the work.

\section{References}

[1] S. P. Zhang, G. D. Fu and Z.W.Zhang, Gas Production Technology of Low Permeability Gas Reservoir in Ordos Basin , Petroleum Industry Press, Bei Jing, 2014.

[2] X.Q. Ran, A.Q. Li, Sulige Gas Field Development Approach, Petroleum Industry Press, Bei Jing, 2008.

[3] Y. Chen, F. F. Guo, X. H. Bai, Development of High Temperature Pressure Sensor Based on SOI, Instrument Technique and Sensors, 6(2014), 4-6. 